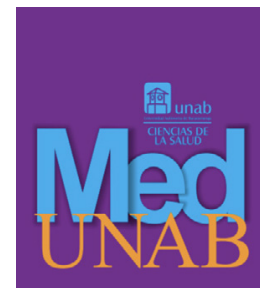

REVISTA DE LA FACULTAD

DE CIENCIAS DE LA SALUD

Vol. 24(2): 279-281, agosto-noviembre 2021 I-SSN $0123-7047$

\title{
Uso indiscriminado del instrumento Zarit en cuidadores de pacientes crónicos no geriátricos ni demenciales
}

Indiscriminate use of the Zarit instrument in caregivers of chronic non-geriatric or dementia patients

Uso indiscriminado do instrumento Zarit em cuidadores de pacientes crônicos não geriátricos ou com demencia

\section{Wilder Omar Zamora-Ormeño, Est. ${ }^{1}$ (D), Dorian Carlo Figueroa-Alvarez, Est. ${ }^{1}$ (D)}

1. Estudiante de Medicina. Universidad Privada San Juan Bautista, Lima, Perú.

Correspondencia. Wilder Omar Zamora-Ormeño. Sector 1, Grupo 2, Manzana B, Lote 1, Villa el Salvador. Email: Wilder.zamora.o@gmail.com

\section{INFORMACIÓN DEL ARTÍCULO:}

Artículo recibido: 18 de diciembre 2020

Artículo aceptado: 30 de junio de 2021

DOI: https://doi.org/10.29375/01237047.4059

Cómo citar: Zamora-Ormeño WO, Figueroa-Alvarez DC. Uso indiscriminado del instrumento Zarit en cuidadores de pacientes crónicos no geriátricos ni demenciales. MedUNAB. 2021;24(2):279-281. doi: https://doi. org/10.29375/01237047.4059

Palabras clave:

Anciano; Demencia; Instrumento Validado de Zarit; Cuidador Informal; Sobrecarga.

Keywords:

Elderly; Dementia; Validated Zarit Instrument; Informal Caregiver; Burden.

Keywords:

Idoso; Demência; Instrumento Zarit Validado; Cuidador Informal; Sobrecarga. 


\section{Estimada editora:}

El artículo publicado en la revista de la Facultad de Ciencias de la Salud por Ángel-García et al., se enfoca en la sobrecarga de los cuidadores informales de pacientes con enfermedades crónicas, tales como hipertensión arterial, diabetes mellitus, cáncer, enfermedades respiratorias, renales y neurológicas. Así mismo, el estudio toma como muestra a cuidadores que se encuentran en edades entre los 18 a 96 años. Por la importancia del artículo para el área de salud, y al revisar su metodología y resultados, se considerará acotar algunos puntos (1).

Los resultados del estudio son importantes, ya que determinan que una mayor competencia y preparación del cuidador generará una menor sobrecarga de estrés en su labor. No obstante, el estudio carece de validez interna al utilizar el instrumento Zarit, el cual no se encuentra validado para medir la sobrecarga en cuidadores de pacientes no geriátricos, ni tampoco para las diversas enfermedades crónicas que presentan los sujetos de cuidado en la muestra del estudio (1).

En primer lugar, el instrumento Zarit fue creado en 1980 con el objetivo principal de evaluar el nivel de estrés de los cuidadores primarios de pacientes geriátricos con cuadros de demencia, este instrumento es un test de 29 preguntas que valoran el estrés psicológico y físico del cuidador; así mismo, evalúa su situación laboral y socioeconómica, y el vínculo con el paciente bajo su cuidado; además, se utiliza solo en cuidadores primarios de pacientes geriátricos y no en todas las enfermedades crónicas, como se menciona en el artículo (2,3). A partir de 1996, esta escala ha sido adaptada y traducida al español por diferentes autores, como Carrasco et al.; el nuevo instrumento Zarit traducido presenta 22 ítems y tiene unos puntos de corte de "no sobrecarga" hasta 46-47 puntos, "sobrecarga leve" hasta 56 puntos y "sobrecarga intensa" si es mayor a 56 (3). La confiabilidad de la herramienta Zarit presenta un coeficiente alfa de Cronbach $=0.91$. Para el estudio de validez de constructor se comprobó una correlación positiva entre el estrés del cuidador y su condición de salud mental calculado con el test de salud general. En el análisis de estadística del instrumento Zarit se llegó a la conclusión de que es un herramienta validada y segura para medir los grados de sobrecarga relativa de cuidadores de adultos mayores con problemas de trastornos psicogeriátricos $(2,4)$. Sin embargo, el Zarit sigue siendo una herramienta válida solo para medir niveles de sobrecarga subjetiva en cuidadores de ancianos que padecen cuadros de demencia u otras afectaciones, pero no se recomienda su uso generalizado, como bien lo menciona Crespo y Rivas (3). Por ende, lo correcto es aplicar este instrumento creado y validado para el grupo etario de pacientes geriátricos, lo cual entra en disonancia al verlo ejecutado en la muestra de la investigación con pacientes entre los 18 a 90 años. Además debemos recordar que utilizar herramientas no validadas para un grupo etario específico puede terminar arrojando datos equivocados y exponer el trabajo a errores metodológicos $(1,5)$.

En segundo lugar, el instrumento Zarit pierde validez con el manejo de cuidadores de pacientes crónicos que no presenten demencia o enfermedades terminales, como lo mencionan Crespo y Rivas (3), quienes concluyen que, si bien desde su creación en 1980 por Zarit et al., la herramienta Zarit Caregiver Burden Interview se ha ido validando y modificando con el paso del tiempo por diferentes autores, mencionando el caso de Gort en el año 2003, el cual extiende la exclusividad de este test hacia los cuidadores de pacientes con demencia $\mathrm{y}$ enfermedades crónicas terminales, $\mathrm{y}$ en el caso de Gutiérrez-Maldonado et al. en el 2005, que amplían la validez de este cuestionario para cuidadores de pacientes con esquizofrenia, como también la escala Zarit reducida para cuidados paliativos por Gort et al en el 2005 (3), esta escala reducida para cuidados paliativos logra un índice de Gra-Qol de 55.5\%, mucho menor en comparación a la escala de Zarit de Carrasco et al en 1996, que obtuvo un índice de Gra-Qol de 90.9\%; el índice de Gra-Qol es un indicador del nivel de desarrollo de una herramienta de medición y su utilidad global basándose en criterios sobre la Calidad de Vida Relacionada con la Salud (4), Esta escala de Zarit española de Carrasco et al fue una adaptación y traducción de la escala original de Zarit de 1983 y validada para cuidadores de pacientes gerontes con demencia similar a la original; por lo que, no se debería generalizar en una misma muestra a todos los cuidadores de cualquier enfermedad con la herramienta Zarit, ya que existen otros instrumentos previamente validados, con un mejor índice Gra-Qol y que encajan mejor con las diferentes patologías y variables epidemiológicas que presentan los cuidadores y sus pacientes, y no como se ha encasillado a todas las patologías crónicas con el mismo instrumento en la metodología del artículo de Ángel-García et al. $(1,4)$.

Para finalizar, es imprescindible que se tome conciencia del uso indiscriminadodel instrumento Zarit en cuidadores de pacientes crónicos no geriátricos ni demenciales, debido a que futuros profesionales e investigadores podrían optar por continuar en esta línea de investigación utilizando instrumentos no validados para su población de estudio. Por ello, se sugiere ampliar o validar la prueba de Zarit para los casos crónicos de enfermedades en general y no solo para trastornos psicogeriátricos; así mismo, validarla para pacientes no geriátricos. También 
se podría buscar aplicar otros instrumentos ya validados y que presentan una mayor utilidad para la población estudiada, u otros que busquen factores como ansiedad, actitud, ánimo y signos de alarma de vulnerabilidad para que los cuidadores reciban una atención oportuna a fin de no terminar en cuadros graves de estrés, burnout o situaciones indeseables; de esta manera, el instrumento podrá tener una repercusión más clínica. Adicionalmente, se deja en evidencia la carencia de nuevos instrumentos orientados a la prevención y promoción de la salud mental en distintos grupos etarios.

\section{Financiación}

Ninguna declarada por los autores.

\section{Conflicto de intereses}

Ninguno declarado por los autores.

\section{Contribución de los autores}

Los autores participaron en la concepción, recolección de información y redacción del artículo.

\section{Referencias}

1. Del Ángel-García JE, León-Hernández RC, MéndezSantos G, Peñarrieta-De Córdova I, Flores-Barrios F. Relación entre sobrecarga y competencias del cuidar en cuidadores informales de personas con enfermedades crónicas. MedUNAB. 2020;23(2):23341. doi: https://doi.org/10.29375/01237047.3878

2. Martos A, Cardila F, Barragán AB, Pérez-Fuentes $\mathrm{MC}$, Molero MM y Gázquez JJ. Instrumento para evaluar la sobrecarga del cuidador familiar de personas con demencia. Eur J Investig Health Psychol Educ. 2015;5(2):281-92. https://doi.org/10.3390/ ejihpe 5020026

3. Crespo María, Rivas Ma Teresa. La evaluación de la carga del cuidador: una revisión más allá de la escala de Zarit. Clínica y Salud. 2015 Mar; 26(1): 9-15. https://dx.doi.org/10.1016/j.clysa.2014.07.002.

4. Delgado-González E, González-Esteban MP, Ballesteros-Álvaro AM, Pérez-Alonso J, MediavillaME, Aragón-Posadas R, et al. Existen instrumentos válidos para medir el síndrome del cuidador familiar, una revista sistémica de la literatura. España: Junta de Castilla y León, Consejería de Sanidad;
2009. Disponible en: http://envejecimiento.csic.es/ documentos/documentos/delgado-instrumentos-01. pdf

5. Alpuche VJ, Ramos Del Rio B, Rojas Russell ME, Figueroa López CG. Validez de la escala de carga de Zarit en una muestra de cuidadores primarios informales. México: Psicología y Salud; 2008. Disponible en: https://www.redalyc.org/ pdf/291/29118209.pdf 